\title{
Synthesis of Functionalized Oxaphosphaphenanthrenes and Chromenes via Multicomponent Reactions of Trivalent Phosphorus Nucleophiles
}

\author{
Zinatossadat Hossaini and Faramarz Rostami-Charati
}

\begin{abstract}
Stable derivatives of oxaphosphaphenanthrenes were prepared using multicomponent reactions of dialkyl acetylenedicarboxylate with 3-bromo-2-naphthol in the presence of trimethyl or triphenyl phosphite in good yields. Chromene derivatives were produced by using triethyl phosphite and dialkyl acetylenedicarboxylate in the presence of $\mathrm{OH}$-acids in excellent yields.
\end{abstract}

Index Terms-One-pot reactions, acetylenic compounds; chromenes, trimethyl phosphate, Triphenyl phosphite.

\section{INTRODUCTION}

Phosphorus compounds containing $\mathrm{P}-\mathrm{C}$ bonds are not mostly abundant in nature but they have diverse biological activity and have attracted significant synthetic and pharmacological interest [1], [2]. Besides precious applications, their use in the construction of the unsafe compounds sarin, soman, and VX-type chemical warfare agents (CWAs) is of note [3]. Phosphonates have important applications in flame retardancy [4], [5], organic synthesis [6], and biological applications [2], [7]. Also, phosphonates have been used as substitutes of the corresponding esters and acids of high biological activity [8], [9] and as suitable probes for designing antibodies on the basis of transition state models. A large number of methods have appeared describing novel syntheses of organophosphorus compounds [10]-[13]. In this research another class of synthesized compounds is chromenes. Chromenes have attracted substantial attention due to their biological activity and their presence in a diversity of significant natural products [14]. Hence, we describe herein the reaction of dialkyl acetylenedicarboxylate with a trivalent phosphorus nucleophile such as trimethyl phosphite, triethyl phosphite, or triphenyl phosphite in the presence of $\mathrm{OH}$-acids.

\section{RESUlt AND DISSCUSSION}

The reaction of dialkyl acetylenedicarboxylate, 3-bromo-2-naphthol and trimethyl or triphenyl phosphite leads to 3-bromo-4H-1-oxa- phosphaphenanthrene-3,4dicarboxylate derivatives 4 in excellent yields [13] (Fig. 1).

Manuscript received April 4, 2014; revised June 23, 2014.

Zinatossadat Hossaini is with the Department of Chemistry, Qaemshahr Branch, Islamic Azad University, P. O. Box 163, Qaemshahr, Iran (corresponding author: Zinatossadat Hossaini; e-mail: zshossaini@yahoo.com).

Faramarz Rostami-Charati is with the Department of Chemistry, Facualty of Science, Gonbad Kavous University, P. O. Box 163, Gonbad, Iran.

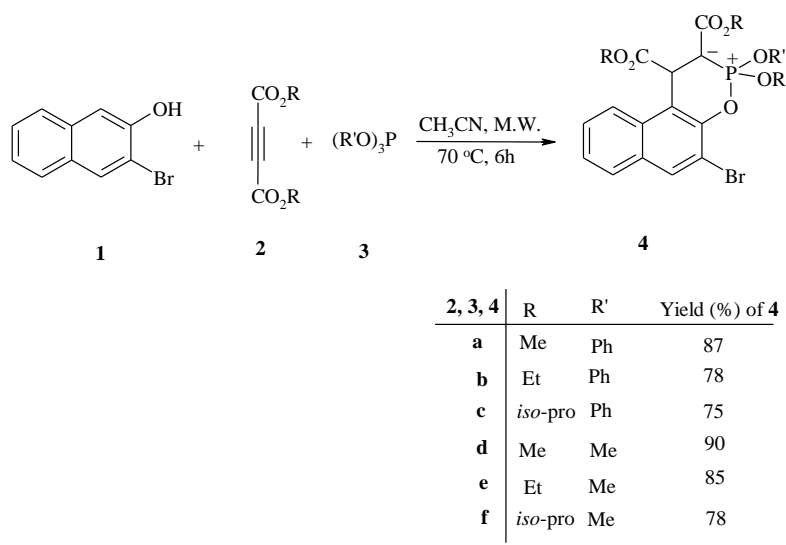

Fig. 1. Reaction of activated acetylene, 3-bromo-2-naphthol and trimethyl or triphenyl phosphite.

The structures of $4 \mathrm{a}-4 \mathrm{f}$ were decided on the basis of their ${ }^{1} \mathrm{H},{ }^{13} \mathrm{C}$, and ${ }^{31} \mathrm{P}$ NMR spectra, IR spectra, elemental analyses, and mass spectrometric data. The ${ }^{1} \mathrm{H}$ NMR spectrum of $4 \mathrm{a}$ in $\mathrm{CDCl}_{3}$ shows two singlets for methoxy protons at 3.25 and $3.82 \mathrm{ppm}$ and one doublets at $\delta=5.54\left({ }^{3} J_{\mathrm{PH}}=27.6 \mathrm{~Hz}\right)$, for the methine proton, along with multiplets at $\delta=6.85-9.12$ for the aromatic protons. The presence of an ylide ester group in 4a stabilizes by the observation of strong low-frequency carbonyl absorption at $1665 \mathrm{~cm}^{-1}$ in the IR spectrum [15]. The other ester carbonyl absorption in $4 \mathrm{a}$ appears at $1723 \mathrm{~cm}^{-1}$. The ${ }^{13} \mathrm{C}$ NMR spectrum consisted characteristic carbonyl resonances at $\delta=167.6\left(\mathrm{~d},{ }^{2} J_{\mathrm{PC}}=16.8 \mathrm{~Hz}\right)$, and $174.5 \mathrm{ppm}$ clearly, whereas the ylide carbon atom displays resonances at $\delta=42.0\left(\mathrm{~d},{ }^{1} J_{\mathrm{PC}}=232 \mathrm{~Hz}\right) \mathrm{ppm}$. The observed ${ }^{1} J_{\mathrm{CP}}$ values are typical of an $\alpha$-ylide ester [16]. The ${ }^{31} \mathrm{P}$ NMR signal was found at $\delta=42.4 \mathrm{ppm}$.

Although the mechanism of this reaction has not been established, a plausible rationalization can be advanced to explain product formation (Fig. 2). On the basis of phosphorus nucleophiles chemistry [11], [17], it is reasonable to presume that ylide 4 results from initial addition of the phosphite to the activated acetylenic compounds and following protonation of the reactive 1:1 adduct, followed by attack of carbon atom of the anion of 3-bromo-2-naphthol 6 to cation 5 to generate ylide 7 which isomerises under the reaction conditions employed to produce the oxaphosphorane 8. Elimination of ROH from 8 leads to product 4 .

The reaction between triethyl phosphite 9, dialkyl acetylenedicarboxylate 2 and 3-bromo-2-naphthol 1 quantitatively generate product 10 (Fig. 3).

The IR spectrum of 10a exhibited the ester and lactone carbonyl groups at 1715 and $1758 \mathrm{~cm}^{-1}$ respectively. The ${ }^{1} \mathrm{H}$ 
NMR spectrum of 10a showed a doublet doublet at $\delta=2.85$ $\left({ }^{2} J_{\mathrm{HH}}=15.4 \mathrm{~Hz},{ }^{3} J_{\mathrm{HH}}=7.2 \mathrm{~Hz}\right)$ for one of the methylene protons. The other methylene proton displayed a doublet at $\delta$ $=3.28\left({ }^{2} J_{\mathrm{HH}}=15.4 \mathrm{~Hz}\right) \mathrm{ppm}$. The methine proton showed a doublet at $\delta=4.64\left({ }^{3} J_{\mathrm{HH}}=7.3 \mathrm{~Hz}\right) \mathrm{ppm}$. The coupling constants monitord for this AMX system are reliable with a conformation in which the $\mathrm{H}-\mathrm{C}-\mathrm{C}-\mathrm{H}$ dihedral angles for the $\mathrm{CH}-\mathrm{CH}_{2}$ moiety are expected to be about $90^{\circ}$ and $30^{\circ}$ [15]. The ${ }^{13} \mathrm{C}$ NMR spectrum of $10 \mathrm{a}$ displayed 15 distinct resonances in agreement with the proposed structure.
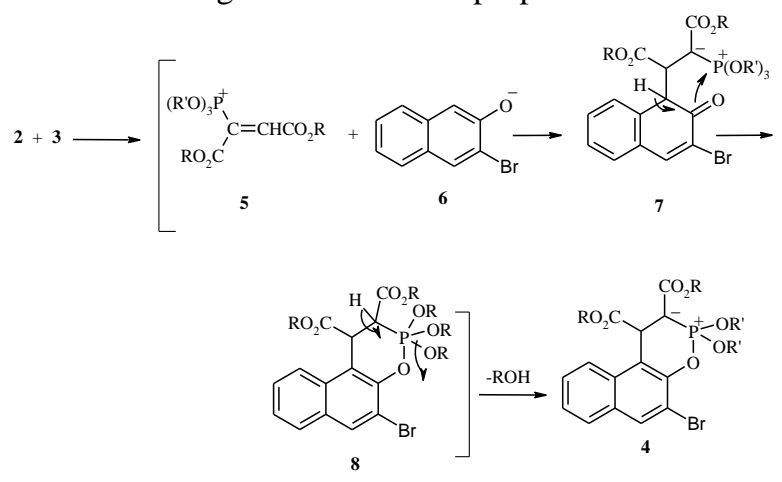

Fig. 2. Proposed mechanism for the formation of 4 .

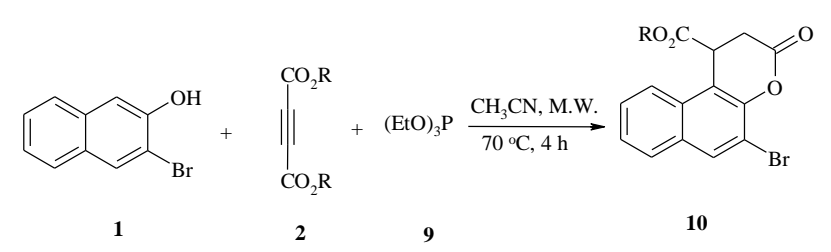

$$
\begin{array}{rl|lc}
\mathbf{2 , 1 0} & \mathrm{R} & \text { Yield (\%) of } \mathbf{1 0} \\
\hline \mathbf{a} & \mathrm{Me} & 90 \\
\mathbf{b} & \mathrm{Et} & 85 \\
\mathbf{c} & { }^{t} \mathrm{Bu} & 78 \\
\text { Fig. 3. Reaction of triethyl phosphite, activated acetylenes and }
\end{array}
$$
3-bromo-2-naphthol.

A possible mechanism for the formation of compound 10 is showed in Fig. 4. The oxaphosphorane 13 is formed in similar steps shown for oxaphosphorane 8 in Fig. 2. However, since the ethoxide anion is a weaker living group, cleavage of the phosphorus-oxygen bond of the 3-bromo-2-naphthol residue become favorable, giving dialkyl succinate 16 . Lactonization of this hydroxy-ester gave product 10 .
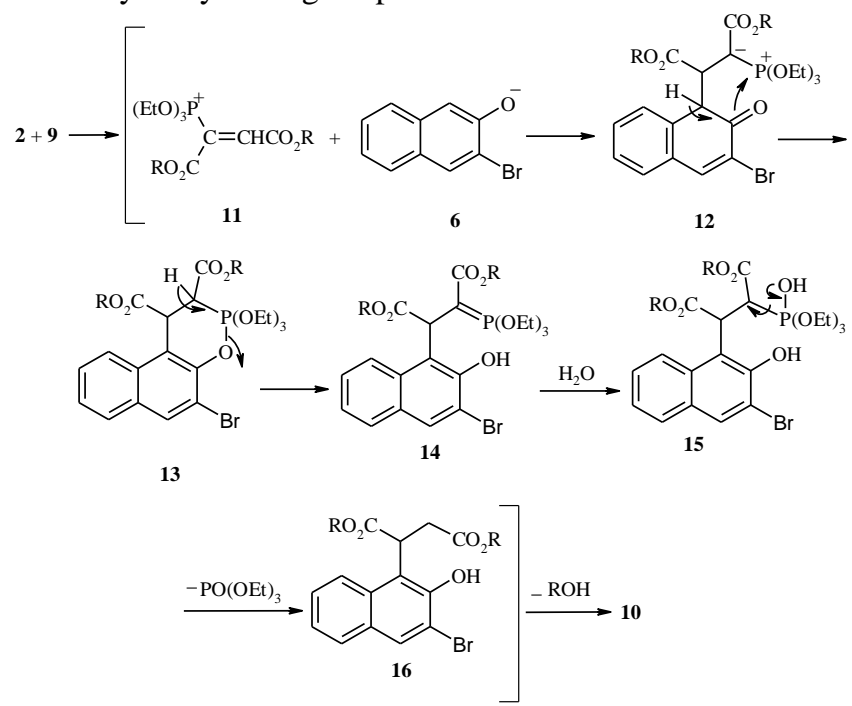

Fig. 4. Proposed mechanism for the formation of 10.

\section{CONCLUSION}

In conclusion, we found that the reaction of activated acetylenic compounds with trimethyl phosphite, triethyl phosphite or triphenyl phosphite in the presence of 3-bromo-2-naphthol or 4-hydroxycumarin leads to a facile synthesis of some functionalized oxaphosphaphenanthrenes and chromenes under microwave conditions without using any catalyst. These reactions were performed in a lab type microwave (in a type of MICROSYNTH from Mylestone Company) system and used microwave irradiation as green source energy for synthesis of heterocyclic compounds.

\section{EXPERIMENTAL}

Melting points were measured on an Electrothermal 9100 aparatus. Elemental analyses for the $\mathrm{C}, \mathrm{H}$, and $\mathrm{N}$ were performed using a Heraeus CHN-O-Rapid analyzer. Mass spectra were recorded on a FINNIGAN-MATT 8430 spectrometer operating at an ionization potential of $70 \mathrm{eV}$. IR spectra were measured on a Shimadzu IR-460 spectrometer. ${ }^{1} \mathrm{H},{ }^{13} \mathrm{C}$, and ${ }^{31} \mathrm{P}$ spectra were measured with a BRUKER DRX-500 AVANCE spectrometer at 500.1, 125.8, and 202.4 $\mathrm{MHz}$, respectively. ${ }^{1} \mathrm{H},{ }^{13} \mathrm{C}$, and ${ }^{31} \mathrm{P}$ spectra were obtained for solutions in $\mathrm{CDCl}_{3}$ using TMS as internal standard or $85 \%$ $\mathrm{H}_{3} \mathrm{PO}_{4}$ as external standard. All the chemicals used in this work were purchased from Fluka (Buchs, Switzerland) and are used without further purification.

General procedure for preparation of compounds 4 .

To a magnetically stirred solution of dialkyl acethylenedicarboxylate $2(2 \mathrm{mmol})$ and 3-bromo-2-naphthol $1(2 \mathrm{mmol})$ in $20 \mathrm{~cm}^{3} \mathrm{CH}_{3} \mathrm{CN}$ was added triphenyl phosphite or trimethyl phosphite 3 (2 mmol) under microwave conditions (In power of $800 \mathrm{w}$ and $\mathrm{T}=70^{\circ} \mathrm{C}$ ). The reaction mixture was then stirred for $6 \mathrm{~h}$. After completion of reaction (monitored by TLC), the mixture of reaction was purified by preparative TLC on silica gel column chromatography (Merck 230-400 mesh) using n-hexane-EtOAc as eluent to give compound 4.

Dimethyl 2,2-diphenoxy-3-bromo-4H-1-oxa-phospha phenanthrene-3,4-dicarboxylate (4a).

White powder, m.p. $162-64{ }^{\circ} \mathrm{C}, 1.00 \mathrm{~g}$, yield $87 \%$. IR (KBr) $\left(v_{\max } / \mathrm{cm}^{-1}\right): 1665$, and $1723 \mathrm{~cm}^{-1}$. Anal. Calcd for $\mathrm{C}_{28} \mathrm{H}_{22} \mathrm{BrO}_{7} \mathrm{P}$ (581.35): C, 57.85; H, 3.81. Found: C, 57.78; H, $3.76 \%$. ${ }^{1} \mathrm{H}$ NMR $\left(500 \mathrm{MHz}, \mathrm{CDCl}_{3}\right): \delta 3.25(3 \mathrm{H}, \mathrm{s}, \mathrm{MeO})$, $3.82(3 \mathrm{H}, \mathrm{s}, \mathrm{MeO}), 5.54\left(1 \mathrm{H}, \mathrm{d},{ }^{3} J_{\mathrm{HP}}=27.6 \mathrm{~Hz}, \mathrm{CH}\right)$, 6.85-9.12 (15 H, m, $15 \mathrm{CH}) .{ }^{13} \mathrm{C}$ NMR (125.7 MHz, $\left.\mathrm{CDCl}_{3}\right)$ : $\delta 42.0\left(\mathrm{~d},{ }^{1} J_{\mathrm{CP}}=232 \mathrm{~Hz}, \mathrm{C}\right), 42.4\left(\mathrm{~d},{ }^{2} J_{\mathrm{CP}}=8.5 \mathrm{~Hz}, \mathrm{CH}\right), 50.4$ (MeO), $52.2(\mathrm{OMe}), 120.7\left(\mathrm{~d},{ }^{3} J_{\mathrm{CP}} 5.2 \mathrm{~Hz}, 2 \mathrm{CH}\right), 121.0(\mathrm{~d}$, $\left.{ }^{3} J_{\mathrm{PC}} 9.6 \mathrm{~Hz}, \mathrm{C}\right), 121.7\left(\mathrm{~d},{ }^{3} J_{\mathrm{PC}} 4.6 \mathrm{~Hz}, 2 \mathrm{CH}\right), 124.3(\mathrm{CH})$, $125.7(\mathrm{CH}), 126.2(\mathrm{CH}), 126.5(\mathrm{CH}), 127.6(\mathrm{CH}), 128.5(\mathrm{CH})$, $130.0(\mathrm{~m}, 4 \mathrm{CH}), 130.4(\mathrm{CH}), 131.3(\mathrm{C}), 131.8(\mathrm{C}), 132.2(\mathrm{C})$, $148.5\left(\mathrm{~d}^{2} J_{\mathrm{PC}} 9.2 \mathrm{~Hz}, \mathrm{C}\right), 150.2(\mathrm{~m}, 2 \mathrm{C}), 167.6\left(\mathrm{~d}^{2} J_{\mathrm{PC}} 16.8 \mathrm{~Hz}\right.$, $\mathrm{C}=\mathrm{O}), 174.5(\mathrm{C}=\mathrm{O}) .{ }^{31} \mathrm{P}$ NMR $\left(202 \mathrm{MHz}, \mathrm{CDCl}_{3}\right): \delta 42.4$.

Diethyl 2,2-diphenoxy-3-bromo-4H-1-oxa-phospha phenanthrene Z-3,4-dicarboxylate (4b).

Pale yellow powder, m.p. $170-172{ }^{\circ} \mathrm{C}, 0.95 \mathrm{~g}$, yield $78 \%$. IR $(\mathrm{KBr})\left(v_{\max } / \mathrm{cm}^{-1}\right): 1670$, and $1728 \mathrm{~cm}^{-1}$. Anal. Calcd for $\mathrm{C}_{30} \mathrm{H}_{26} \mathrm{BrO}_{7} \mathrm{P}$ (609.41): C, 59.13; H, 4.30. Found: C, 59.26; H, 4.38\%. ${ }^{1} \mathrm{H} \mathrm{NMR}\left(500 \mathrm{MHz}, \mathrm{CDCl}_{3}\right): \delta 1.32\left(3 \mathrm{H}, \mathrm{t},{ }^{3} J_{\mathrm{HH}}=7.4\right.$, 
$\mathrm{Me}), 1.37\left(3 \mathrm{H}, \mathrm{t},{ }^{3} J_{\mathrm{HH}}=7.3, \mathrm{Me}\right), 4.22\left(2 \mathrm{H}, \mathrm{q},{ }^{3} J_{\mathrm{HH}}=7.4\right.$, $\left.\mathrm{CH}_{2} \mathrm{O}\right), 4.28\left(2 \mathrm{H}, \mathrm{q},{ }^{3} \mathrm{~J}_{\mathrm{HH}}=7.4, \mathrm{CH}_{2} \mathrm{O}\right), 5.58\left(1 \mathrm{H}, \mathrm{d},{ }^{3} \mathrm{~J}_{\mathrm{HP}}=\right.$ $28.0 \mathrm{~Hz}, \mathrm{CH}), 6.82-9.10(15 \mathrm{H}, \mathrm{m}, 15 \mathrm{CH}) .{ }^{13} \mathrm{C} \mathrm{NMR}(125.7$ $\left.\mathrm{MHz}, \mathrm{CDCl}_{3}\right): \delta 13.8(\mathrm{Me}), 14.2(\mathrm{Me}), 42.2\left(\mathrm{~d},{ }^{1} J_{\mathrm{CP}}=230 \mathrm{~Hz}\right.$, C), $42.5\left(\mathrm{~d},{ }^{2} J_{\mathrm{CP}}=8.7 \mathrm{~Hz}, \mathrm{CH}\right), 61.7\left(\mathrm{CH}_{2} \mathrm{O}\right), 62.3\left(\mathrm{CH}_{2} \mathrm{O}\right)$, $121.2\left(\mathrm{~d},{ }^{3} J_{\mathrm{CP}} 5.8 \mathrm{~Hz}, 2 \mathrm{CH}\right), 121.4\left(\mathrm{~d},{ }^{3} J_{\mathrm{PC}} 10.4 \mathrm{~Hz}, \mathrm{C}\right), 122.3$ $\left(\mathrm{d},{ }^{3} J_{\mathrm{PC}} 5.5 \mathrm{~Hz}, 2 \mathrm{CH}\right), 124.8(\mathrm{CH}), 126.0(\mathrm{CH}), 126.5(\mathrm{CH})$, $127.2(\mathrm{CH}), 127.8(\mathrm{CH}), 129.2(\mathrm{CH}), 130.4(\mathrm{~m}, 4 \mathrm{CH}), 130.7$ (CH), $131.6(\mathrm{C}), 132.2(\mathrm{C}), 132.7(\mathrm{C}), 149.0\left(\mathrm{~d}^{2} J_{\mathrm{PC}} 9.5 \mathrm{~Hz}\right.$, C), $150.8(\mathrm{~m}, 2 \mathrm{C}), 168.0\left(\mathrm{~d}^{2} J_{\mathrm{PC}} 16.2 \mathrm{~Hz}, \mathrm{C}=\mathrm{O}\right), 174.8(\mathrm{C}=\mathrm{O})$ ${ }^{31} \mathrm{P}$ NMR (202 MHz, $\left.\mathrm{CDCl}_{3}\right): \delta 42.5$.

Diisopropyl 2,2-diphenoxy-3-bromo-4H-1-oxa-phospha phenanthrene-3,4-dicarboxylate (4c).

Pale Yellow powder, m.p. $182-184{ }^{\circ} \mathrm{C}, 0.90 \mathrm{~g}$, yield $75 \%$. IR $(\mathrm{KBr})\left(v_{\max } / \mathrm{cm}^{-1}\right): 1674$, and $1735 \mathrm{~cm}^{-1}$. Anal. Calcd for $\mathrm{C}_{32} \mathrm{H}_{30} \mathrm{BrO}_{7} \mathrm{P}$ (637.46): C, 60.29; H, 4.74. Found: $\mathrm{C}, 60.18 ; \mathrm{H}$, 4.65\%. ${ }^{1} \mathrm{H}$ NMR $\left(500 \mathrm{MHz}, \mathrm{CDCl}_{3}\right): \delta=1.35\left(6 \mathrm{H}, \mathrm{d},{ }^{3} J_{\mathrm{HH}}=\right.$ $\left.6.5 \mathrm{~Hz}, 2 \mathrm{CH}_{3}\right), 1.42\left(6 \mathrm{H}, \mathrm{d},{ }^{3} J_{\mathrm{HH}}=6.8 \mathrm{~Hz}, 2 \mathrm{CH}_{3}\right), 5.28-5.36$ $(1 \mathrm{H}, \mathrm{m}, \mathrm{CH}), 5.38-5.44(1 \mathrm{H}, \mathrm{m}, \mathrm{CH}), 5.62\left(1 \mathrm{H}, \mathrm{d},{ }^{3} J_{\mathrm{HP}}=\right.$ $28.4 \mathrm{~Hz}, \mathrm{CH}), 6.85-9.15(15 \mathrm{H}, \mathrm{m}, 15 \mathrm{CH}) .{ }^{13} \mathrm{C} \mathrm{NMR}(125.7$ $\left.\mathrm{MHz}, \mathrm{CDCl}_{3}\right): \delta 21.6\left(2 \mathrm{CH}_{3}\right), 22.3\left(2 \mathrm{CH}_{3}\right), 42.6\left(\mathrm{~d},{ }^{1} J_{\mathrm{CP}}=\right.$ $234.5 \mathrm{~Hz}, \mathrm{C}), 42.8\left(\mathrm{~d},{ }^{2} J_{\mathrm{CP}}=9.3 \mathrm{~Hz}, \mathrm{CH}\right), 68.8\left(C \mathrm{HMe}_{2}\right), 70.2$ $\left(C \mathrm{HMe}_{2}\right), 121.6\left(\mathrm{~d},{ }^{3} J_{\mathrm{CP}} 6.0 \mathrm{~Hz}, 2 \mathrm{CH}\right), 122.0\left(\mathrm{~d},{ }^{3} J_{\mathrm{PC}} 10.5 \mathrm{~Hz}\right.$, C), $122.5\left(\mathrm{~d},{ }^{3} J_{\mathrm{PC}} 5.8 \mathrm{~Hz}, 2 \mathrm{CH}\right), 125.2(\mathrm{CH}), 126.7(\mathrm{CH})$, $127.3(\mathrm{CH}), 127.6(\mathrm{CH}), 128.4(\mathrm{CH}), 129.5(\mathrm{CH}), 130.8(\mathrm{~m}, 4$ $\mathrm{CH}), 131.2(\mathrm{CH}), 132.3(\mathrm{C}), 132.8(\mathrm{C}), 133.4(\mathrm{C}), 149.4(\mathrm{~d}$ $\left.{ }^{2} J_{\mathrm{PC}} 9.8 \mathrm{~Hz}, \mathrm{C}\right), 151 .(\mathrm{m}, 2 \mathrm{C}), 168.6\left(\mathrm{~d}^{2} J_{\mathrm{PC}} 15.8 \mathrm{~Hz}, \mathrm{C}=\mathrm{O}\right)$, $175.5(\mathrm{C}=\mathrm{O}) .{ }^{31} \mathrm{P}$ NMR $\left(202 \mathrm{MHz}, \mathrm{CDCl}_{3}\right): \delta 43.2$.

Dimethyl 2,2-dimethoxy-3-bromo-4H-1-oxa-phospha phenanthrene-3,4-dicarboxylate (4d).

White crystals, m.p. $132-134{ }^{\circ} \mathrm{C}, 0.64$ g, yield $90 \%$. IR $(\mathrm{KBr})\left(v_{\max } / \mathrm{cm}^{-1}\right): 1650$, and $1727 \mathrm{~cm}^{-1}$. Anal. Calcd for $\mathrm{C}_{18} \mathrm{H}_{18} \mathrm{BrO}_{7} \mathrm{P}$ (457.21): C, 47.29; H, 3.97. Found: C, 47.34; H, 4.03\%. ${ }^{1} \mathrm{H}$ NMR (500 MHz, $\left.\mathrm{CDCl}_{3}\right): \delta 3.62(3 \mathrm{H}, \mathrm{s}, \mathrm{MeO})$, $3.75(3 \mathrm{H}, \mathrm{s}, \mathrm{MeO}), 3.78$ ( $\left.3 \mathrm{H}, \mathrm{d}^{3} J_{\mathrm{PH}} 13.5 \mathrm{~Hz}, \mathrm{MeO}\right), 3.97$ (3 $\left.\mathrm{H}, \mathrm{d}^{3} J_{\mathrm{PH}} 13.5 \mathrm{~Hz}, \mathrm{MeO}\right), 5.67\left(1 \mathrm{H}, \mathrm{d}^{3} J_{\mathrm{HP}} 31.7 \mathrm{~Hz}, \mathrm{CH}\right), 7.45$ $\left(1 \mathrm{H}, \mathrm{t},{ }^{3} J_{\mathrm{HH}} 9.4 \mathrm{~Hz}, \mathrm{CH}\right), 7.62\left(1 \mathrm{H}, \mathrm{t},{ }^{3} J_{\mathrm{HH}} 9.4 \mathrm{~Hz}\right), 7.82(1 \mathrm{H}$, $\left.\mathrm{d}^{3} J_{\mathrm{HH}} 9.6 \mathrm{~Hz}, \mathrm{CH}\right), 7.87\left(1 \mathrm{H}, \mathrm{d}^{3} J_{\mathrm{HH}} 9.6 \mathrm{~Hz}, \mathrm{CH}\right), 8.12(1 \mathrm{H}$, $\mathrm{s}, \mathrm{CH}) .{ }^{13} \mathrm{C}$ NMR $\left(125.7 \mathrm{MHz}, \mathrm{CDCl}_{3}\right): \delta 39.4\left(\mathrm{~d},{ }^{1} J_{\mathrm{CP}} 225.2\right.$ $\mathrm{Hz}, \mathrm{C}), 41.6\left(\mathrm{~d},{ }^{2} J_{\mathrm{CP}} 9.4 \mathrm{~Hz}, \mathrm{CH}\right), 50.4(\mathrm{MeO}), 52.3(\mathrm{MeO})$, $55.6\left(\mathrm{~d},{ }^{2} J_{\mathrm{PC}} 6.4 \mathrm{~Hz}, \mathrm{P}-\mathrm{OMe}\right), 55.7\left(\mathrm{~d},{ }^{2} J_{\mathrm{PC}} 6.4 \mathrm{~Hz}, \mathrm{P}-\mathrm{OMe}\right)$, $118.4\left(\mathrm{~d},{ }^{3} J_{\mathrm{PC}} 6.8 \mathrm{~Hz}, \mathrm{C}\right), 121.4\left(\mathrm{~d},{ }^{3} J_{\mathrm{PC}} 9.5 \mathrm{~Hz}, \mathrm{C}\right), 124.6$ $(\mathrm{CH}), 125.5(\mathrm{CH}), 127.4(\mathrm{CH}), 128.6(\mathrm{CH}), 130.0(\mathrm{CH})$, $131.4(\mathrm{C}), 131.7$ (C), $149.2\left(\mathrm{~d}^{2} J_{\mathrm{PC}} 8.4 \mathrm{~Hz}, \mathrm{C}-\mathrm{O}\right), 169.6\left(\mathrm{~d}^{2} J_{\mathrm{PC}}\right.$ $18.4 \mathrm{~Hz}, \mathrm{C}=\mathrm{O}), 175.2(\mathrm{C}=\mathrm{O}) .{ }^{31} \mathrm{P}$ NMR $\left(202 \mathrm{MHz} \mathrm{CDCl}_{3}\right): \delta$ 42.8 .

Diethyl 2,2-dimethoxy-3-bromo-4H-1-oxa-phospha phenanthrene-3,4-dicarboxylate (4e).

Pale yellow powder, m.p. $136-138{ }^{\circ} \mathrm{C}, 0.82 \mathrm{~g}$, yield $85 \%$. IR $(\mathrm{KBr})\left(v_{\max } / \mathrm{cm}^{-1}\right): 1656$, and $1735 \mathrm{~cm}^{-1}$. Anal. Calcd for $\mathrm{C}_{20} \mathrm{H}_{22} \mathrm{BrO}_{7} \mathrm{P}$ (485.27): C, 49.50; H, 4.57. Found: C, 49.62; H, 4.67\%. ${ }^{1} \mathrm{H} \mathrm{NMR}\left(500 \mathrm{MHz}, \mathrm{CDCl}_{3}\right): \delta 1.28\left(3 \mathrm{H}, \mathrm{t},{ }^{3} J_{\mathrm{HH}}=7.2\right.$, $\mathrm{Me}), 1.34\left(3 \mathrm{H}, \mathrm{t},{ }^{3} J_{\mathrm{HH}}=7.2, \mathrm{Me}\right), 4.18\left(2 \mathrm{H}, \mathrm{q},{ }^{3} J_{\mathrm{HH}}=7.2\right.$, $\left.\mathrm{CH}_{2} \mathrm{O}\right), 4.25\left(2 \mathrm{H}, \mathrm{q},{ }^{3} J_{\mathrm{HH}}=7.2, \mathrm{CH}_{2} \mathrm{O}\right), 5.63\left(1 \mathrm{H}, \mathrm{d}^{3} J_{\mathrm{HP}} 32.0\right.$ $\mathrm{Hz}, \mathrm{CH}), 7.52\left(1 \mathrm{H}, \mathrm{t},{ }^{3} J_{\mathrm{HH}} 9.8 \mathrm{~Hz}, \mathrm{CH}\right), 7.67\left(1 \mathrm{H}, \mathrm{t},{ }^{3} J_{\mathrm{HH}} 9.7\right.$ $\mathrm{Hz}), 7.86\left(1 \mathrm{H}, \mathrm{d}^{3} J_{\mathrm{HH}} 10.2 \mathrm{~Hz}, \mathrm{CH}\right), 7.93\left(1 \mathrm{H}, \mathrm{d}^{3} J_{\mathrm{HH}} 10.2 \mathrm{~Hz}\right.$, $\mathrm{CH}), 8.08(1 \mathrm{H}, \mathrm{s}, \mathrm{CH}) .{ }^{13} \mathrm{C} \mathrm{NMR}\left(125.7 \mathrm{MHz}, \mathrm{CDCl}_{3}\right): \delta 13.8$ (Me), $14.3(\mathrm{Me}), 39.6\left(\mathrm{~d},{ }^{1} J_{\mathrm{CP}} 226.4 \mathrm{~Hz}, \mathrm{C}\right), 42.0\left(\mathrm{~d},{ }^{2} J_{\mathrm{CP}} 9.8\right.$ $\mathrm{Hz}, \mathrm{CH}), 56.2\left(\mathrm{~d},{ }^{2} J_{\mathrm{PC}} 7.2 \mathrm{~Hz}, \mathrm{P}-\mathrm{OMe}\right), 56.5\left(\mathrm{~d},{ }^{2} J_{\mathrm{PC}} 7.2 \mathrm{~Hz}\right.$, P-OMe), $61.4\left(\mathrm{CH}_{2} \mathrm{O}\right), 62.3\left(\mathrm{CH}_{2} \mathrm{O}\right), 119.2\left(\mathrm{~d},{ }^{3} \mathrm{~J}_{\mathrm{PC}} 7.4 \mathrm{~Hz}, \mathrm{C}\right)$, $121.8\left(\mathrm{~d},{ }^{3} J_{\mathrm{PC}} 10.3 \mathrm{~Hz}, \mathrm{C}\right), 125.2(\mathrm{CH}), 125.7(\mathrm{CH}), 127.9$ $(\mathrm{CH}), 129.2(\mathrm{CH}), 130.4(\mathrm{CH}), 131.8(\mathrm{C}), 132.2(\mathrm{C}), 150.2(\mathrm{~d}$ $\left.{ }^{2} J_{\mathrm{PC}} 8.2 \mathrm{~Hz}, \mathrm{C}-\mathrm{O}\right), 170.4\left(\mathrm{~d}^{2} J_{\mathrm{PC}} 18.6 \mathrm{~Hz}, \mathrm{C}=\mathrm{O}\right), 175.8(\mathrm{C}=\mathrm{O})$. ${ }^{31} \mathrm{P}$ NMR $\left(202 \mathrm{MHz}, \mathrm{CDCl}_{3}\right): \delta 43.2$.

Diisopropyl 2,2-dimethoxy-3-bromo-4H-1-oxa-phospha phenanthrene-3,4-dicarboxylate (4f).

Yellow powder, m.p. $145-147{ }^{\circ} \mathrm{C}, 0.80$ g, yield $78 \%$. IR $(\mathrm{KBr})\left(v_{\max } / \mathrm{cm}^{-1}\right): 1657$ and $1736 \mathrm{~cm}^{-1}$. Anal. Calcd for $\mathrm{C}_{22} \mathrm{H}_{26} \mathrm{BrO}_{7} \mathrm{P}$ (513.32): C, 51.48; H, 5.11. Found: $\mathrm{C}, 51.54 ; \mathrm{H}$, $5.18 \% .{ }^{1} \mathrm{H} \mathrm{NMR}\left(500 \mathrm{MHz}, \mathrm{CDCl}_{3}\right): \delta=1.35\left(6 \mathrm{H}, \mathrm{d},{ }^{3} J_{\mathrm{HH}}=\right.$ $\left.7.0 \mathrm{~Hz}, 2 \mathrm{CH}_{3}\right), 1.44\left(6 \mathrm{H}, \mathrm{d},{ }^{3} J_{\mathrm{HH}}=7.0 \mathrm{~Hz}, 2 \mathrm{CH}_{3}\right), 5.32-5.40$ $(1 \mathrm{H}, \mathrm{m}, \mathrm{CH}), 5.44-5.53(1 \mathrm{H}, \mathrm{m}, \mathrm{CH}), 5.67\left(1 \mathrm{H}, \mathrm{d}^{3} J_{\mathrm{HP}} 31.8\right.$ $\mathrm{Hz}, \mathrm{CH}), 7.55\left(1 \mathrm{H}, \mathrm{t},{ }^{3} J_{\mathrm{HH}} 10.2 \mathrm{~Hz}, \mathrm{CH}\right), 7.72\left(1 \mathrm{H}, \mathrm{t},{ }^{3} J_{\mathrm{HH}}\right.$ $10.5 \mathrm{~Hz}), 7.92\left(1 \mathrm{H}, \mathrm{d}^{3} J_{\mathrm{HH}} 10.5 \mathrm{~Hz}, \mathrm{CH}\right), 7.98\left(1 \mathrm{H}, \mathrm{d}^{3} J_{\mathrm{HH}}\right.$ $10.6 \mathrm{~Hz}, \mathrm{CH}), 8.05(1 \mathrm{H}, \mathrm{s}, \mathrm{CH}) .{ }^{13} \mathrm{C} \mathrm{NMR}(125.7 \mathrm{MHz}$, $\left.\mathrm{CDCl}_{3}\right): \delta 21.7\left(2 \mathrm{CH}_{3}\right), 22.5\left(2 \mathrm{CH}_{3}\right), 40.2\left(\mathrm{~d},{ }^{1} J_{\mathrm{CP}} 226.8 \mathrm{~Hz}\right.$, C), $42.5\left(\mathrm{~d},{ }^{2} J_{\mathrm{CP}} 9.8 \mathrm{~Hz}, \mathrm{CH}\right), 56.4\left(\mathrm{~d},{ }^{2} J_{\mathrm{PC}} 7.0 \mathrm{~Hz}, \mathrm{P}-\mathrm{OMe}\right)$, $56.7\left(\mathrm{~d},{ }^{2} J_{\mathrm{PC}} 7.0 \mathrm{~Hz}, \mathrm{P}-\mathrm{OMe}\right), 68.7\left(C \mathrm{HMe}_{2}\right), 70.4\left(C \mathrm{HMe}_{2}\right)$, $119.5\left(\mathrm{~d},{ }^{3} J_{\mathrm{PC}} 6.8 \mathrm{~Hz}, \mathrm{C}\right), 122.0\left(\mathrm{~d},{ }^{3} J_{\mathrm{PC}} 9.7 \mathrm{~Hz}, \mathrm{C}\right), 124.8$ $(\mathrm{CH}), 125.3(\mathrm{CH}), 128.2(\mathrm{CH}), 129.4(\mathrm{CH}), 130.8(\mathrm{CH})$, $132.3(\mathrm{C}), 132.7$ (C), $150.6\left(\mathrm{~d}^{2} J_{\mathrm{PC}} 8.5 \mathrm{~Hz}, \mathrm{C}-\mathrm{O}\right), 170.6\left(\mathrm{~d}^{2} J_{\mathrm{PC}}\right.$ $17.8 \mathrm{~Hz}, \mathrm{C}=\mathrm{O}), 176.3(\mathrm{C}=\mathrm{O}) .{ }^{31} \mathrm{P} \mathrm{NMR}\left(202 \mathrm{MHz}, \mathrm{CDCl}_{3}\right): \delta$ 43.4 .

General procedure for preparation of compounds 10 .

To a magnetically stirred solution of dialkyl acethylenedicarboxylate $2(2 \mathrm{mmol})$ and 3-bromo-2-naphthol $1(2 \mathrm{mmol})$ in $20 \mathrm{~cm}^{3} \mathrm{CH}_{3} \mathrm{CN}$ was added triethyl phosphate 9 ( $2 \mathrm{mmol}$ ) under microwave conditions (In power of $800 \mathrm{~W}$ and $\mathrm{T}=70^{\circ} \mathrm{C}$ ). The reaction mixture was then stirred for $4 \mathrm{~h}$. After completion of reaction (monitored by TLC), the mixture of reaction was purified by preparative TLC on silica gel column chromatography (Merck 230-400 mesh) using $n$-hexane-EtOAc as eluent to give compound 10 .

Methyl

5-bromo-3-oxo-2,3-dihydro-1H-benzo[f]chromene-1-carbox ylate (10a).

White powder, m.p. $158-160{ }^{\circ} \mathrm{C}, 0.60 \mathrm{~g}$, yield $90 \%$. IR $(\mathrm{KBr})\left(v_{\max } / \mathrm{cm}^{-1}\right): 1715$, and $1758 \mathrm{~cm}^{-1}$. Anal. Calcd for $\mathrm{C}_{15} \mathrm{H}_{11} \mathrm{BrO}_{4}$ (335.15): C, 53.76; H, 3.31. Found: C, 53.72; H, $3.42 \% .{ }^{1} \mathrm{H}$ NMR $\left(500 \mathrm{MHz}, \mathrm{CDCl}_{3}\right): \delta 2.85\left(1 \mathrm{H}, \mathrm{dd}^{2} J_{\mathrm{HH}}\right.$ $\left.15.4 \mathrm{~Hz}^{3} J_{\mathrm{HH}} 7.2 \mathrm{~Hz}, \mathrm{HCH}\right), 3.28\left(1 \mathrm{H}, \mathrm{d}^{2} J_{\mathrm{HH}} 15.4 \mathrm{~Hz}, \mathrm{HCH}\right)$, $3.72(3 \mathrm{H}, \mathrm{s}, \mathrm{MeO}), 4.64\left(1 \mathrm{H}, \mathrm{d}^{3} J_{\mathrm{HH}} 7.3 \mathrm{~Hz}, \mathrm{CH}\right), 7.52(1 \mathrm{H}$, $\left.\mathrm{t},{ }^{3} J_{\mathrm{HH}} 7.8 \mathrm{~Hz}, \mathrm{CH}\right), 7.60\left(1 \mathrm{H}, \mathrm{t},{ }^{3} J_{\mathrm{HH}} 8.2 \mathrm{~Hz}, \mathrm{CH}\right), 7.75(1 \mathrm{H}$, $\left.\mathrm{d}^{3} J_{\mathrm{HH}} 8.2 \mathrm{~Hz}, \mathrm{CH}\right), 7.83(1 \mathrm{H}, \mathrm{s}, \mathrm{CH}), 8.35\left(1 \mathrm{H},{ }^{3} J_{\mathrm{HH}} 8.7 \mathrm{~Hz}\right.$, $\mathrm{CH}) .{ }^{13} \mathrm{C} \mathrm{NMR}\left(125.7 \mathrm{MHz}, \mathrm{CDCl}_{3}\right): \delta 31.6\left(\mathrm{CH}_{2}\right), 38.2(\mathrm{CH})$, $52.8(\mathrm{MeO}), 112.6(\mathrm{C}), 114.3(\mathrm{C}), 123.3(\mathrm{CH}), 125.7(\mathrm{CH})$, $128.3(\mathrm{CH}), 128.8(\mathrm{CH}), 131.4(\mathrm{CH}), 131.9(\mathrm{C}), 132.3(\mathrm{C})$, $150.6(\mathrm{C}), 166.5(\mathrm{C}=\mathrm{O}), 172.4(\mathrm{C}=\mathrm{O})$. MS, $m / z(\%): 335\left(\mathrm{M}^{+}\right.$, 15), 304 (48), 207 (68), 128 (76), 31 (100).

Ethyl 5-bromo-3-oxo-2,3-dihydro-1H-benzo[f]chromene -1-carboxylate (10b).

White powder, m.p. $165-167{ }^{\circ} \mathrm{C}, 0.60 \mathrm{~g}$, yield $85 \%$. IR $(\mathrm{KBr})\left(v_{\max } / \mathrm{cm}^{-1}\right): 1718$, and $1755 \mathrm{~cm}^{-1}$. Anal. Calcd for $\mathrm{C}_{16} \mathrm{H}_{13} \mathrm{BrO}_{4}$ (349.18): C, 55.04; H, 3.75. Found: C, 55.15; H, $3.83 \% .{ }^{1} \mathrm{H} \mathrm{NMR}\left(500 \mathrm{MHz}, \mathrm{CDCl}_{3}\right): \delta 1.32\left(3 \mathrm{H}, \mathrm{t},{ }^{3} J_{\mathrm{HH}}=7.4\right.$, $\mathrm{Me}), 2.87\left(1 \mathrm{H}, \mathrm{dd}^{2} J_{\mathrm{HH}} 15.6 \mathrm{~Hz}^{3} J_{\mathrm{HH}} 7.2 \mathrm{~Hz}, \mathrm{HCH}\right), 3.34(1 \mathrm{H}$, $\left.\mathrm{d}^{2} J_{\mathrm{HH}} 15.4 \mathrm{~Hz}, \mathrm{HCH}\right), 4.25\left(2 \mathrm{H}, \mathrm{q},{ }^{3} J_{\mathrm{HH}} 7.2 \mathrm{~Hz}, \mathrm{CH}_{2} \mathrm{O}\right), 4.65$ $\left(1 \mathrm{H}, \mathrm{d}^{3} J_{\mathrm{HH}} 7.2 \mathrm{~Hz}, \mathrm{CH}\right), 7.58\left(1 \mathrm{H}, \mathrm{t},{ }^{3} J_{\mathrm{HH}} 7.6 \mathrm{~Hz}, \mathrm{CH}\right), 7.60$ $\left(1 \mathrm{H}, \mathrm{t},{ }^{3} J_{\mathrm{HH}} 7.8 \mathrm{~Hz}, \mathrm{CH}\right), 7.78\left(1 \mathrm{H}, \mathrm{d}^{3} J_{\mathrm{HH}} 8.3 \mathrm{~Hz}, \mathrm{CH}\right), 7.85$ $(1 \mathrm{H}, \mathrm{s}, \mathrm{CH}), 8.37\left(1 \mathrm{H},{ }^{3} \mathrm{~J}_{\mathrm{HH}} 8.5 \mathrm{~Hz}, \mathrm{CH}\right) .{ }^{13} \mathrm{C}$ NMR $(125.7$ 
$\left.\mathrm{MHz}, \mathrm{CDCl}_{3}\right): \delta 13.7(\mathrm{Me}), 32.0\left(\mathrm{CH}_{2}\right), 38.4(\mathrm{CH}), 61.6$ $\left(\mathrm{CH}_{2} \mathrm{O}\right), 113.2(\mathrm{C}), 114.7(\mathrm{C}), 123.6(\mathrm{CH}), 126.4(\mathrm{CH}), 128.7$ $(\mathrm{CH}), 129.4(\mathrm{CH}), 131.6(\mathrm{CH}), 132.0(\mathrm{C}), 132.7(\mathrm{C}), 151.3$ (C), $166.8(\mathrm{C}=\mathrm{O}), 172.6(\mathrm{C}=\mathrm{O})$.

Tert-butyl 5-bromo-3-oxo-2,3-dihydro-1H-benzo[f] chromene-1-carboxylate (10c).

White powder, m.p. $178-180{ }^{\circ} \mathrm{C}, 0.59$ g, yield $78 \%$. IR $(\mathrm{KBr})\left(v_{\max } / \mathrm{cm}^{-1}\right): 1716$, and $1762 \mathrm{~cm}^{-1}$. Anal. Calcd for $\mathrm{C}_{18} \mathrm{H}_{17} \mathrm{BrO}_{4}$ (377.23): C, 57.31; H, 4.54. Found: C, 57.38; H, 4.62\%. ${ }^{1} \mathrm{H} \mathrm{NMR}\left(500 \mathrm{MHz}, \mathrm{CDCl}_{3}\right): \delta 1.65\left(9 \mathrm{H}, \mathrm{s}, 3 \mathrm{CH}_{3}\right)$, $2.92\left(1 \mathrm{H}, \mathrm{dd}^{2} J_{\mathrm{HH}} 16.2 \mathrm{~Hz}{ }^{3} J_{\mathrm{HH}} 7.4 \mathrm{~Hz}, \mathrm{HCH}\right), 3.27(1 \mathrm{H}, \mathrm{d}$ $\left.{ }^{2} J_{\mathrm{HH}} 15.4 \mathrm{~Hz}, \mathrm{HCH}\right), 4.67\left(1 \mathrm{H}, \mathrm{d}^{3} J_{\mathrm{HH}} 7.5 \mathrm{~Hz}, \mathrm{CH}\right), 7.63(1 \mathrm{H}$, $\left.\mathrm{t},{ }^{3} J_{\mathrm{HH}} 7.7 \mathrm{~Hz}, \mathrm{CH}\right), 7.68\left(1 \mathrm{H}, \mathrm{t},{ }^{3} J_{\mathrm{HH}} 8.7 \mathrm{~Hz}, \mathrm{CH}\right), 7.82(1 \mathrm{H}$, $\left.\mathrm{d}^{3} J_{\mathrm{HH}} 8.3 \mathrm{~Hz}, \mathrm{CH}\right), 7.86(1 \mathrm{H}, \mathrm{s}, \mathrm{CH}), 8.37\left(1 \mathrm{H},{ }^{3} J_{\mathrm{HH}} 8.6 \mathrm{~Hz}\right.$, $\mathrm{CH}) .{ }^{13} \mathrm{C}$ NMR $\left(125.7 \mathrm{MHz}, \mathrm{CDCl}_{3}\right): \delta 28.7\left(3 \mathrm{CH}_{3}\right), 32.4$ $\left(\mathrm{CH}_{2}\right), 38.5(\mathrm{CH}), 53.2(\mathrm{MeO}), 83.5\left(\mathrm{CMe}_{3}\right), 113.4(\mathrm{C}), 115.2$ (C), $124.0(\mathrm{CH}), 126.2(\mathrm{CH}), 128.5(\mathrm{CH}), 129.2(\mathrm{CH}), 131.8$ $(\mathrm{CH}), 132.3(\mathrm{C}), 132.6(\mathrm{C}), 151.0(\mathrm{C}), 167.4(\mathrm{C}=\mathrm{O}), 173.5$ $(\mathrm{C}=\mathrm{O})$.

\section{REFERENCES}

[1] P. C. Hinkle and R. E. McCarty, "Primary active sodium transport, oxygen consumption, and ATP: Coupling and regulation," Sci. Am., 1978, vol. 238, p. 104-123.

[2] R. Engel, "Phosphonates as analogues of natural phosphates," Chem. Rev. 1977, vol. 77, p. 349.

[3] R. M. Black, R. J. Clark, R. W. Read, and M. T. J. Reid, "Determination of the isoelectric point of the capillary capillary electrophoresis, Application to plastic capillaries," J. Chromatogr. A 1994, vol. 662, pp. 369-373.

[4] E. S. Papazoglou, Handbook of Building Materials for Fire Protection, C. A. Harper, Ed., McGraw-Hill, New York, 2004.

[5] E. D. Weil, In Kirk-Othmer Encyclopedia of Chemical Technology, John Wiley, New York, 1993, vol. 4, p. 976.

[6] B. E. Maryanoff and A. B. Reitz, "The Wittig olefination reaction and modifications involving phosphoryl-stabilized carbanions. Stereochemistry, mechanism, and selected synthetic aspects," Chem. Rev., 1989, vol. 89, p. 863.

[7] G. A. Freeman, J. L. Rideout, W. H. Miller, and J. E. Reardon, “3'-Azido-3',5'-dideoxythymidine-5'-methylphosphonic acid diphosphate: synthesis and HIV-1 reverse transcriptase inhibition," $J$. Med. Chem., 1992, vol. 35, p. 3192.

[8] B. Kaboudin and R. Nazari, "Synthesis of phosphorothioates using thiophosphate salts," Tetrahedron Lett., 2001, vol. 42, p. 8211.

[9] D. Y. Kim and D. Y. Rhie, "Asymmetric synthesis of aziridine 2-phosphonates and azirinyl phosphonates from enantiopure sulfinimines," Tetrahedron, 1997, vol. 53, pp. 13603-13608.

[10] G. M. Kosolapoff and L. Maier, Organic Phosphorus Compounds, Willey-Interscience, a division of John Wiley \& Sons, Inc., 1972.
[11] H. R. Hudson, "Primary, secondary and tertiary phosphines, polyphosphines and heterocyclic organophosphorus (III) Compounds," in The Chemistry of Organophosphorus Compounds, F. R. Hantely, Wiley Ed., New York, vol. 1, pp. 386-472, 1990.

[12] R. Engel, Synthesis of Carbon-Phosphorus Bonds, CRC Press: Boca Raton, FL, 1998.

[13] I. Yavari, M. Anary-Abbasinejad, and Z. Hossaini, Org. Biomol. Chem., 2003, vol. 1, pp. 560 .

[14] K. C. Nicolaou, J. A. Pfefferkorn, A. J. Roecker, G. Q. Cao, S. Barluenga, and H. J. Mitchell, "Natural product-like combinatorial libraries based on privileged structures. 1. general principles and solid-phase synthesis of benzopyrans," J. Am. Chem. Soc. 2000, vol. 122, pp. 9939-9953.

[15] R. M. Silverstein, C. G. Bassler, and T. C. Morrill, Spectroscopic Identification of Organic Compounds, 5th ed., Wiley, New York, 1992.

[16] R. Engle, Synthesis of Carbon-Phosphorus Bond, CRC Press, Boca Raton, FL, 1988.

[17] J. I. G. Cadogan, Organophosphorus Reagents in Organic Synthesis, Academic Press, New York, 1977.

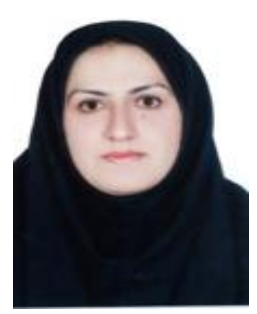

Zinatossadat Hossaini was born in 1976, Tehran Iran. She received her B.S. degree in chemistry in 2000 from Alzahra University, Tehran, Iran, M.S degree in organic chemistry in 2003 from Tarbiat Modares University, Tehran, Iran, and her Ph.D. degree in organic chemistry in 2008 from Tarbiat Modares University under the supervision of Dr. Issa Yavari, Tehran, Iran. Currently, she is an assistant professor of organic chemistry at the Chemistry Department, Faculty of Science, Islamic Azad University, Qaemshahr, Iran. She owns membership of Nokhbegan National Institute (since 2006) and Young Selected Academic People of Iran. Her research interests and activities include synthesis and characterization of new organic compounds especially heterocyclic compound under various conditions such as multicomponent, one-pot, tandem, MW, solvent-free, thermal, and catalytic conditions.

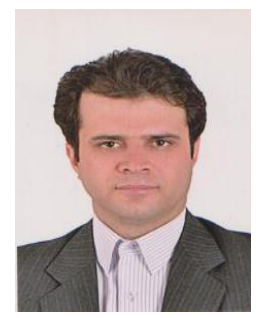

Faramarz Rostami-Charati was born in 1976, Babol, Iran. He received his B.S. degree in chemistry in 2000 from Esfahan University, Esfahan, Iran, M.S. degree in organic chemistry in 2003 from Sistan and Balochestan University, Sistan and Balochestan, Iran, and his Ph.D. degree in organic chemistry in 2007 from Sistan and Balochestan University under the supervision of Dr Maghsoudlo, Sistan and Balochestan, Iran. Currently, he is an assistant professor of organic chemistry at the Chemistry Department, Faculty of Science, Gonbad Kavous University, Gonbad, Iran. His research interests and activities include phythochemistry and synthesis of heterocyclic compound under various conditions such as multicomponent, one-pot, tandem, MW, solvent-free, thermal, and catalytic conditions. 